\title{
COMORBIDITY OF CHRONIC DISEASES IN GENERAL PRACTICE
}

\author{
F. G. Schellevis, ${ }^{1,2, *}$ J. VAN DeR Velden, ${ }^{3}$ E. VAN de LisdonK, ${ }^{2}$ \\ J. TH. M. VAN EIJK ${ }^{1}$ and C. VAN WeEL ${ }^{2}$ \\ 'Department of General Practice and Nursing Home Medicine, Vrije Universiteit, Amsterdam, \\ ${ }^{2}$ Department of General Practice, University of Nijmegen and ${ }^{3}$ Netherlands Institute of Primary \\ Health Care (NIVEL), Utrecht, The Netherlands
}

(Received in revised form 30 November 1992)

\begin{abstract}
With the increasing number of elderly people in The Netherlands the prevalence of chronic diseases will rise in the next decades. It is recognized in general practice that many older patients suffer from more than one chronic disease (comorbidity). The aim of this study is to describe the extent of comorbidity for the following diseases: hypertension, chronic ischemic heart disease, diabetes mellitus, chronic nonspecific lung disease, osteoarthritis. In a general practice population of 23,534 persons, 1989 patients have been identified with one or more chronic diseases. Only diseases in agreement with diagnostic criteria were included. In persons of 65 and older $23 \%$ suffer from one or more of the chronic diseases under study. Within this group $15 \%$ suffer from more than one of the chronic diseases. Osteoarthritis and diabetes mellitus are the diseases with the highest rate of comorbidity. Comorbidity restricts the external validity of results from single-disease intervention studies and complicates the organization of care.
\end{abstract}

Comorbidity Chronic diseases General practice Prevalence

\section{INTRODUCTION}

The morbidity pattern in general practice is well-documented, particularly in The Netherlands and in the U.K., where the fixed practice population allows for population-based description [1-4].

General practice covers its own clinical spectrum as has been demonstrated previously [5]. Chronic diseases are an important feature of this clinical spectrum: hypertension, chronic ischemic heart disease, diabetes mellitus, chronic nonspecific lung disease, and osteoarthritis all have a prevalence above 10 per 1000 and are mostly managed in general practice $[3,4,6,7]$. In the near future the number of elderly people

*All correspondence should be addressed to: F.G Schellevis, Department of General Practice and Nursing Home Medicine, v.d. Boechorststraat 7, 1081 BT Amsterdam, The Netherlands. will increase in The Netherlands. As a consequence, the prevalence of chronic diseases will rise.

These changes in morbidity pattern will influence the daily work in general practice. Standards and guidelines for proper diagnosis, treatment and management of chronic diseases are crucial for maintaining the quality of care. Intervention studies provide an essential basis for adequate treatment and prevention. Most of such studies analyze the effects of intervention on a single disease. General practitioners (GPs), however, recognize that their patients often suffer from more than one chronic disease. As a generalist the GP, alone or in cooperation with the specialist, deals with all diseases of a patient. As the natural course and the therapeutic interventions of one disease will influence the co-existing second (or even third) disease [8], comorbidity diminishes the practical value of 
single-disease standards for treatment and management, derived from single-disease trials. We found no publications on the frequency of comorbidity in general practice populations. The aim of this study is to describe the extent of comorbidity of chronic diseases in gencral practice in The Netherlands: how many of the patients are under care for more than one of the following, most common, chronic diseases: hypertension, chronic ischemic heart disease, diabetes mellitus, chronic nonspecific lung disease, and osteoarthritis?

The findings indicate the prevalence of these problems and thus contribute to our insight in disease clustering [9].

\section{METHODS}

This study is part of a larger research on the prevalence of chronic diseases in general practice, and of the effects of systematic surveillance on the quality of care.

\section{General practices and population}

Seven general practices (15 GPs) participated in the study. The practices covered 23,534 persons at the start of the data collection (1 January 1988). An age/sex register of the practices was completed with the help of trained students. The number of persons of 65 years and over is $3 \%$ less than in the entire country ( 9.3 vs $12.5 \%$-Table 1$)$. For that reason all results are presented for two subpopulations: that of persons below 65 and that of persons of 65 and over. In other characteristics the practice population differs only marginally from the entire population [10].

Table 1. Age and sex distribution of the population of the general practices studied $(N=23,534)$ compared to the population of The Netherlands $(N=14,714,948) 1$ January 1988

\begin{tabular}{lcc}
\hline & $\begin{array}{c}\text { Practice } \\
\text { population } \\
(\%)\end{array}$ & $\begin{array}{c}\text { The Netherlands } \\
(\%)\end{array}$ \\
\hline $0-4$ & 5.4 & 6.1 \\
$5-14$ & 12.9 & 12.4 \\
$15-24$ & 17.3 & 16.8 \\
$25-44$ & 35.8 & 31.9 \\
$45-64$ & 19.3 & 20.4 \\
$\geqslant 65$ & 9.3 & 12.5 \\
Male & 48.8 & 49.4 \\
Female & 51.2 & 50.6 \\
\hline
\end{tabular}

\section{Case identification}

The participating GPs identified all patients known to them with the following diseases:

-hypertension

-diabetes mellitus

-chronic ischemic heart disease (CIHD) (angina pectoris, previous myocardial infarction, coronary sclerosis)

chronic nonspecific lung disease (asthma, chronic bronchitis, emphysema (CNSLD)

-osteoarthritis of hip and/or knee.

Identification took place on the occasion of a consultation, a repeat prescription or another administrative reason for visiting the practice during the first 3 months. Finally, the GP reviewed systematically all patient records to identify diseases of patients who were not seen.

This process of identification resulted in a total number of 1989 patients with 2295 diseases (cases).

\section{Application of diagnostic criteria}

The GP provided retrospective data of the medical history from the patients' records in relation to the diagnostic procedures applied in diagnosing the chronic disease, regardless of whether the diagnosis was made in general practice or by a medical specialist. These data were compared with the inclusion criteria of the International Classification of Health Problems in Primary Care $[10,11]$. Only the cases (diseases) meeting these inclusion criteria were used for analysis.

\section{Analysis}

Comorbidity of chronic diseases is defined as the "point-prevalent concurrence" of the studied diseases known to the participating GPs. Point-prevalence reflects the number of diseases in the population at 1 January 1988. Comorbidity was analyzed on patient level by means of the multiple response technique in SPSS [12]. Comorbidity is expressed as the number of the studied diseases per patient, the mean number of diseases per patient in each disease category, and the proportion of patients with at least one of the other diseases. Due to the cross-sectional measurement and the method of presentation, patients with comorbidity appear in each of the disease categories that apply to them and are therefore counted more than once (e.g. a patient with diabetes and with hypertension appears in the hypertension group as well as in the diabetes 
Table 2. Point-prevalence in persons $<65(N=21,349)$ and $\geqslant 65(N=2185)$ of 5 chronic diseases per 1000 persons in the general practices studied

\begin{tabular}{|c|c|c|c|c|}
\hline & \multicolumn{2}{|c|}{$<65$} & \multicolumn{2}{|c|}{$\geqslant 65$} \\
\hline & Prevalence & $95 \% \mathrm{CI}$ & Prevalence & $95 \% \mathrm{CI}$ \\
\hline Hypertension & 18.5 & 16.620 .3 & 89.7 & $\begin{array}{lll}78.1 & 102.0\end{array}$ \\
\hline CIHD & 7.5 & $6.4-8.7$ & 75.1 & $64.4-86.9$ \\
\hline Diabetes mellitus & 4.3 & $3.4-5.1$ & 33.0 & $25.8-41.3$ \\
\hline CNSLD & 12.2 & $10.8-13.7$ & 43.5 & $35.3-52.9$ \\
\hline $\begin{array}{l}\text { Osteoarthritis } \\
\text { hip/knee }\end{array}$ & 1.7 & $1.1-2.2$ & 29.3 & $22.6-37.3$ \\
\hline
\end{tabular}

category). Proportions and means are presented with the $95 \%$ confidence intervals $(\mathrm{CI})$.

\section{RESULTS}

Our definition of comorbidity is strongly connected with the prevalences of the studied diseases. Table 2 lists the point-prevalences on 1 January 1988 of the diseases meeting the inclusion criteria. The prevalence of most diseases is high in persons over 65 . Hypertension is the most frequent of the studied diseases. Table 3 shows the distribution of the number of the diseases per patient. In the younger subgroup there are few persons known to have one of the studied diseases, and comorbidity occurs in only $0.3 \%$ of these persons. Of the persons over 65 years old, more than $75 \%$ are known not to have one of the 5 chronic diseases, but of the older patients who do have one of these diseases, $16 \%$ has more than one chronic disease. Tables 4(A) and (B) show the extent of comorbidity in patients with at least one disease for the two age groups. In patients under 65 years old patients with osteoarthritis have the highest rate of comorbidity. The most frequent second disease in these patients is CNSLD (5 of 36 patients). In diabetics under $65(N=91)$ hypertension is the most frequent second disease $(15 \%)$. In patients of 65 years and older the highest frequency of comorbidity is found in patients with diabetes mellitus. The most frequent second chronic disease in diabetics over

Table 3. Number of studied chronic diseases per person in the population of the general practices studied

\begin{tabular}{lcc}
\hline & $<65$ & $\geqslant 65$ \\
Number of & $(N=21,349)$ & $(N=2185)$ \\
chronic diseases & $(\%)$ & $(\%)$ \\
\hline None & 95.9 & 76.9 \\
One & 3.8 & 19.5 \\
Two & 0.3 & 3.2 \\
Three & $<0.1$ & 0.3 \\
Four & - & $<0.1$ \\
\hline
\end{tabular}

65 is CIHD (22\%), followed by hypertension $(19 \%)$. High rates of comorbidity are also found in patients with osteoarthritis (mostly hypertension and CNSLD) and CIHD (mostly hypertension).

\section{DISCUSSION}

\section{Prevalence of chronic diseases}

This study is based on data obtained from medical records. Generally this leads to an underestimation of the number of cases in the population. Moreover, only cases in agreement with diagnostic criteria were included. the prevalence of hypertension, chronic ischemic heart discase, diabetes mellitus, and chronic nonspecific lung disease is lower than in other Dutch reports from general practice [3, 4]. Compared with data from the U.K., the prevalence of diabetes mellitus is higher, as has been reported by others [13].

Chronic nonspecific lung disease has been identified as an area of diagnostic confusion. Differences in opinion between physicians in the U.K. and The Netherlands exist as to whether asthma, chronic bronchitis and emphysema have the same pathophysiological characteristics, the so-called Dutch hypothesis, which is heavily disputed $[14,15]$. As Dutch GPs are familiar with the diagnostic label of chronic nonspecific lung disease, this term is used in our description of comorbidity.

\section{Extent of comorbidity}

Comorbidity is a quantitatively important phenomenon in patients over 65 with a chronic disease. Most people over $65(77 \%)$ do not suffer from any of the 5 most common chronic diseases, but within the affected group $16 \%$ is known to suffer from at least one other of the 5 chronic diseases studied.

The occurrence by chance of two diseases in one person can be estimated by multiplying the 
Table 4 (A). Extent of comorbidity per disease for patients $<65$, presented as number of chronic diseases per patient group and as fraction of patients with comorbidity per patient group

\begin{tabular}{|c|c|c|c|c|}
\hline & \multirow[b]{2}{*}{$N$} & \multicolumn{2}{|c|}{$\begin{array}{l}\text { Number of } \\
\text { chronic diseases } \\
\text { per patient }\end{array}$} & \multirow{2}{*}{$\begin{array}{l}\text { Fraction of } \\
\text { patients witl } \\
\text { comorbidity } \\
\% \text { of } N\end{array}$} \\
\hline & & Mean & $95 \% \mathrm{CI}$ & \\
\hline Hypertension & 394 & 1.1 & $1.07-1.13$ & 9.6 \\
\hline CIHD & 161 & 1.2 & $1.14-1.28$ & 19.9 \\
\hline Diabetes mellitus & 91 & 1.2 & $1.13-1.33$ & 20.9 \\
\hline CNSLD & 250 & 1.1 & $1.04-1.11$ & 7.6 \\
\hline $\begin{array}{l}\text { Osteoarthritis } \\
\text { hip/knee }\end{array}$ & 36 & 1.3 & $1.12-1.43$ & 27.8 \\
\hline
\end{tabular}

CIHD $=$ chronic ischemic heart disease CNSLD $=$ chronic nonspecific lung disease.

Table 4 (B). Extent of comorbidity per disease for patients $\geqslant 65$, presented as number of chronic diseases per patient group and as fraction of patients with comorbidity per patient group

\begin{tabular}{|c|c|c|c|c|}
\hline & \multirow[b]{2}{*}{$N$} & \multicolumn{2}{|c|}{$\begin{array}{l}\text { Number of } \\
\text { chronic diseases } \\
\text { per patient }\end{array}$} & \multirow{2}{*}{$\begin{array}{c}\text { Fraction of } \\
\text { patients with } \\
\text { comorbidity } \\
\% \text { of } N\end{array}$} \\
\hline & & Mean & $95 \% \mathrm{CI}$ & \\
\hline Hypertension & 196 & 1.3 & $1.18-1.32$ & 21.9 \\
\hline CIHD & 164 & 1.3 & $1.24-1.42$ & 28.0 \\
\hline Diabetes mellitus & 72 & 1.5 & $1.32-1.62$ & 40.3 \\
\hline CNSLD & 93 & 1.3 & $1.18-1.42$ & 24.7 \\
\hline $\begin{array}{l}\text { Osteoarthritis } \\
\text { hip/knee }\end{array}$ & 64 & 1.4 & $1.22-1.50$ & 32.8 \\
\hline
\end{tabular}

prevalences of the separate diseases. The observed comorbidity of the 5 diseases under study is significantly higher. Having a chronic disease apparently means being at higher risk to have a second or even third disease.

By including only diagnoses meeting diagnostic criteria and by disregarding false negative diagnoses, we probably underestimate also the extent of comorbidity. Moreover, these figures are related only to the 5 chronic conditions under study. The rate of comorbidity would have been even larger, if additional diseases, like malignant neoplasms, epilepsy and other neurological diseases, stroke, peripheral vascular disease, peptic ulcer disease, had been considered. On the other hand, by estimating the extent of comorbidity in a general practice setting bias due to the Berkson's fallacy cannot be excluded: patients under care for a chronic disease are at higher risk for detection of diseases than persons who do not receive such care.

Comorbidity is partly the result of a common pathophysiological process or of complications in the natural course of a disease, as is the case for diabetes mellitus and cardiovascular disease [16]. In other cases, comorbidity of chronic diseases is accidental and cannot be explained pathophysiologically.

\section{CONSEQUENCES}

Persons suffer from more than one chronic disease morc frequently than could be expected by chance from the prevalence of the disease in the general population. This is a clinical reality of medical practice with consequences for research and for the organization of daily care.

\section{Research}

Optimal patient care should ideally be based on valid results from clinical trials. In intervention studies, however, patients with comorbidity often are excluded in the selection of a study group, e.g. in the well-known therapeutic trials in hypertension $[17,18]$. This selection restricts the external validity of the results for excluded patient categories, as has recently been described for the elderly and women $[19,20]$. The existence of a second disease complicates the choice of the antihypertensive treatment that is proven to be effective in single disease patients (e.g. diuretics in diabetes, bèta-blockers in lung disease). Strictly speaking, these studies have not proven the effectiveness of antihypertensive treatment in lowering blood pressure and decreasing cardiovascular morbidity and mortality for patients with comorbidity. In intervention studies on treatment of chronic diseases patients 
with comorbidity should be included. In analyzing the data, patients with comorbidity can be handled as a subgroup, or adjustment of the results for comorbidity can be carried out.

The specific combinations of chronic diseases need further exploration, in order to gain more insight into patterns of disease clustering and hypothetically common etiology.

\section{Care}

Systematic surveillance of patients with chronic diseases is essential in order to provide them with optimal care [7]. Patients with more than one chronic disease are at risk of being included in more than one surveillance scheme. This should be recognized when designing surveillance programs, since it would be counter-productive to have patients visit the practice on various different occasions, as a result of following different schemes for each of their diseases. Careful registration of all diseases is conditional not only for organizational reasons but also for the care to be provided.

Chronic diseases are regarded as "the very stuff of general practice" [6]. Proper management of patients with comorbidity of chronic diseases presents a real challenge to the GP.

\section{REFERENCES}

1. Fry J. Common Diseases. Lancaster: MTP Press Limited; 1985: 4th edn.

2. Royal College of General Practitioners, Office of Population Censuses and Surveys and Department of Health and Social Security. Morbidity Statistics from General Practice. Third National Study 1981-1982. London: HMSO; 1986.

3. Hoogen HJM vd, Huygen FJA, Schellekens JWG, Straat JM, Velden HGM vd, Eds. Morbidity Figures from General Practice. Data from Four General Practices 1978-1982. Nijmegen: Nijmegen University
Department of General Practice; 1985.

4. Lamberts $\mathbf{H}$. Morbidity in General Practice. Utrecht: Huisartsenpers; 1984.

5. Fry J. Perspectives on health care. Update 1977; 15: 999.

6. Hasler J. The very stuff of general practice. MacKenzie lecture. J R Coll Gen Pract 1985; 35: 121-127.

7. Hasler J, Schofield Th. Continuing Care. The Management of Chronic Diseases. Oxford: Oxford University Press; 1990: 2nd edn.

8. Feinstein AR. The pre-therapeutic classification of co-morbidity in chronic disease. J Chron Dis 1970; 23: 455-468.

9. Anonymous. Disease clustering: hide or seek? [Editorial]. Lancet 1990; 336: 717-718.

10. Schellevis FG, Lisdonk EH vd, Velden J vd, Eijk JThM v, Weel C v. Validity of diagnoses of chronic diseases in general practice. The application of diagnostic criteria. J Clin Epidemiol 1993; 46: 461-468.

11. Classification Committee of WONCA. International Classification of Health Problems in Primary Care (ICHPPC)-2-Defined. Oxford: Oxford University Press; 1983: 3rd edn.

12. Anonymous. SPSS Version 4.1. Chicago: SPSS Inc; 1990.

13. Reenders K, Nobel E de, Hoogen HJM vd, Rutten GEHM, Weel C v. Diabetes and its longterm complications in general practice. A survey in a well defined population. Fam Pract 1993; in press.

14. Sluiter HJ, Koëter GH, Monchy JGR de, Postma DS, Vries K de, Orie NGM. The Dutch hypothesis (chronic non-specific lung disease) revisited. Eur Respir J 1991; 4: $479-489$

15. Vermeire PA, Pride NB. A "splitting" look at chronic nonspecific lung disease (CNSLD): common features but diverse pathogenesis. Eur Respir J 1991; 4: $490-496$.

16. Kaplan MH, Feinstein AR. The importance of classifying initial comorbidity in evaluating the outcome of diabetes mellitus. J Chron Dis 1974; 27: 387-404.

17. Management Committee. The Australian therapeutic trial in mild hypertension. Lancet 1980; i: 1261-1267.

18. Medical Research Council Working Party. MRC-trial of treatment of mild hypertension: principal results. Br Med J 1985; 291: 97-104.

19. Gurwitz JH, Col NF, Avorn J. The exclusion of the elderly and women from clinical trials in acute myocardial infarction. JAMA 1992; 268: 1417-1422.

20. Wenger NK. Exclusion of the elderly and women from coronary trials. Is their quality of care compromised? [Editorial]. JAMA 1992; 268: 1460-1461. 